\title{
Premières impressions sur l'Exposition universelle de Shanghai
}

La Chine se met en scène

\section{Florence Padovani}

\section{(2) OpenEdition}

Journals

Édition électronique

URL : http://journals.openedition.org/echogeo/12139

DOI : $10.4000 /$ echogeo.12139

ISSN : 1963-1197

Éditeur

Pôle de recherche pour l'organisation et la diffusion de l'information géographique (CNRS UMR 8586)

Référence électronique

Florence Padovani, «Premières impressions sur l'Exposition universelle de Shanghai », EchoGéo [En ligne], Sur le Vif, mis en ligne le 22 novembre 2010, consulté le 13 novembre 2019. URL : http:// journals.openedition.org/echogeo/12139; DOI : 10.4000/echogeo.12139

Ce document a été généré automatiquement le 13 novembre 2019.

\section{(c) (†) $\odot$}

EchoGéo est mis à disposition selon les termes de la licence Creative Commons Attribution - Pas d'Utilisation Commerciale - Pas de Modification 4.0 International 


\title{
Premières impressions sur l'Exposition universelle de Shanghai
}

\author{
La Chine se met en scène
}

Florence Padovani

\section{NOTE DE L'AUTEUR}

L'auteur tient à remercier les relecteurs pour leurs judicieuses remarques qui ont été prises en compte dans la version définitive. Que Messieurs Thierry Husberg et JeanFrançois Cuenot soient aussi remerciés pour leur assistance technique.

Une vaste marche en avant de la foule Idée conduite par l'esprit Légion. La circulation décuplée ayant pour résultat la production et la consommation centuplées ; la multiplication de pains, de miracle, devenue réalité ; les cours d'eau endigués, ce qui empêchera les inondations, et empoissonnés, ce qui produira la vie à bas prix ;

l'industrie engendrant l'industrie, les bras appelant les bras, l'œuvre faite se ramifiant en

innombrables œuvres à faire, un perpétuel recommencement sorti d'un perpétuel achèvement, et, en tout lieu, à toute heure, sous la hache féconde du progrès, l'admirable renaissance des têtes de l'hydre sainte du travail.

Victor Hugo, Introduction au Paris-guide de

l'exposition universelle de 1869, Chapitre I

«L'Avenir »

1 L'Exposition Universelle qui a eu lieu à Shanghai entre le $1^{\text {er }}$ mai et le 31 septembre 2010 est l'aboutissement d'un long processus qui doit marquer la ville d'accueil, mais aussi la 
Chine face au monde et inversement le monde face à la Chine. En 2009, Pékin accueillait les Jeux Olympiques, en 2010 c'est Shanghai qui est mise en avant et en 2011 ce sera Canton qui accueillera les Jeux Asiatiques. Les trois grands pôles économiques et politiques de la Chine sont donc, l'un après l'autre, organisateur de grands rassemblements internationaux ou régionaux visant à montrer le meilleur de la Chine.

2 En ce qui concerne la municipalité de Shanghai, elle célèbre comme l'avait fait dans une certaine mesure l'exposition universelle de Paris en 1869, le progrès réalisé et propose une vision résolument optimiste de l'avenir. Les premières expositions de la fin du $19^{\text {ème }}$ siècle ont montré la modernité telle que l'envisageait alors le monde européen de la "révolution industrielle». Aujourd'hui, plus d'un siècle après et plusieurs crises internationales majeures plus tard, le concept est toujours autant source de dynamisme. A cela plusieurs raisons, d'abord le fait que pour de nouveaux pays qui revendiquent une place sur la scène internationale, l'organisation d'une exposition internationale permet cette reconnaissance à moindre coût politique et idéologique. "L'Expo 2010 Shanghai sera la première exposition universelle enregistrée auprès du Bureau international des expositions (BIE) qui aura lieu dans un pays en voie de développement. ${ }^{1}$ Hormis le Japon qui avait organisé deux expositions universelles en 1970 puis en 2005 à Osaka puis à Aichi, aucun autre pays asiatique n'avait jusqu'alors accueilli l'événement. Plusieurs expositions spécialisées ont été organisées au Japon $(1975,1985,2005)$ et en Corée du Sud $(1993,2012)$. La Chine se devait donc de faire aussi bien que son rival nippon.

\section{Comment organise-t-on une exposition universelle?}

3 Elle répond à des critères rigoureux imposés par le BIE. Cet organisme, basé à Paris, ne fut créé qu'en 1921 alors que la première exposition universelle de l'ère moderne avait eu lieu à Londres en 1851, les différents conflits en Europe retardèrent sa fondation. A ce jour 157 États en sont membres ${ }^{2}$ En tant qu'organisme international qui supervise les différentes expositions, le BIE fournit le cadre réglementaire à ces évènements mondiaux de prestige auxquels participent les pays du monde entier, les organisations internationales, les groupes de la société civile ainsi que les citoyens. La mission du BIE est d'assurer l'intégrité et la qualité des Expos afin qu'elles continuent à éduquer le public et à promouvoir l'innovation au service du progrès humain ${ }^{3}$.

Deux catégories de manifestations sont homologuées par le BIE: les expositions internationales enregistrées (communément appelées expositions universelles) et les expositions internationales reconnues. Elles sont soumises à des règles différentes.

\begin{tabular}{|l|l|l|}
\hline & $\begin{array}{l}\text { Expositions universelles } \\
\text { (Exposition Internationales } \\
\text { Enregistrées) }\end{array}$ & $\begin{array}{l}\text { Expositions Internationales } \\
\text { Reconnues Spécialisées }\end{array}$ \\
\hline Durée & 6 semaines à 6 mois & 3 semaines à 3 mois \\
\hline Fréquence & Tous les 5 ans & Entre 2 expos universelles \\
\hline Thème & Universel & Précis \\
\hline Surface & Illimitée & 25 hectares maximum \\
\hline
\end{tabular}




\begin{tabular}{|l|l|l|}
\hline Aménagement & $\begin{array}{l}\text { Chaque pays participant conçoit et } \\
\text { construit son pavillon }\end{array}$ & $\begin{array}{l}\text { Le pays hôte met des structures à } \\
\text { disposition des participants }\end{array}$ \\
\hline Lieu & $\begin{array}{l}\text { Une ville capitale nationale ou } \\
\text { régionale }\end{array}$ & \\
\hline
\end{tabular}

5 La candidature est déposée par le gouvernement de l'Etat qui souhaite organiser l'événement au plus tôt neuf ans ou au plus tard six ans avant la date d'ouverture. Le dossier est tout d'abord examiné par la Commission Exécutive du BIE qui donne un avis en fonction du thème choisi, de la date, de l'emplacement, de la superficie du site, du nombre de visiteurs attendus et des modalités financières. Dans une seconde étape, le projet est soumis au vote des pays membres du BIE. Un même pays ne peut recevoir d'exposition (toute catégorie confondue) que tous les dix ans.

\section{Le concept de ville mise en scène}

6 «Une exposition universelle se caractérise par la vaste portée du thème choisi qui doit être un sujet d'intérêt et d'actualité pour l'ensemble de l'humanité. $»^{4}$ Le thème retenu par la municipalité de Shanghai «Meilleure Ville, meilleure Vie» est suffisamment généraliste pour englober toute une série d'aspects très différents. Il s'inscrit aussi dans la lignée des expositions précédentes du troisième millénaire: Hanovre (2000) "L'Homme, la nature, la technologie ; économie d'énergie et d'espace ", Aichi - Japon(2005) «La sagesse de la nature », Saragosse (2008) « L'eau et le développement durable », ou futures comme Yeosu - Corée du sud- (2012) «Les océans, les côtes et la lutte contre le réchauffement climatique », Milan (2015) « Nourrir la planète ».

7 Comme le souligne Vincente Gonzalez Locestales, Secrétaire général du BIE ${ }^{5}$ les thématiques sur le développement des villes concernent aujourd'hui $60 \%$ de la population planétaire. En Chine, les métropoles d'importance sont nombreuses et le gouvernement central n'avait que l'embarras du choix. Wuhan, Chongqing, Canton et tant d'autres pouvaient se transformer en vitrine du développement urbain à la chinoise. Si comme le dit Pascal Ory "l'Exposition universelle est toujours un acte politique. Une sorte de garden-party du pouvoir en place qui met en scène sa propagande $»^{6}$, alors elle ne pouvait se dérouler qu'à Shanghai. La métropole s'affirme de plus en plus comme une place financière de premier plan qui fournit des garanties indispensables au niveau international. C'est aussi une ville ouverte sur l'Occident qui redessine son passé au temps des Concessions et le met en valeur. L'Expo 2010 est donc intégrée dans ce double mouvement de modernisation architecturale et de conservation d'une partie du patrimoine urbain.

8 La manifestation eut lieu au cœur de la ville de Shanghai, comme le montre la carte cidessous. La municipalité de Shanghai explicite le concept de ville. Plusieurs usines ont été déplacées pour laisser l'emplacement libre sur les bords du Huangpu. Le fleuve sépare les deux parties de l'exposition: au sud les pavillons par pays et par thèmes; au nord, les pavillons d'entreprises. Grâce aux nouveaux moyens de transports le site est assez proche des différents centres de Shanghai (la place du Peuple, le Bund ou Lu Jiazui). 
Carte 1 - Carte de localisation

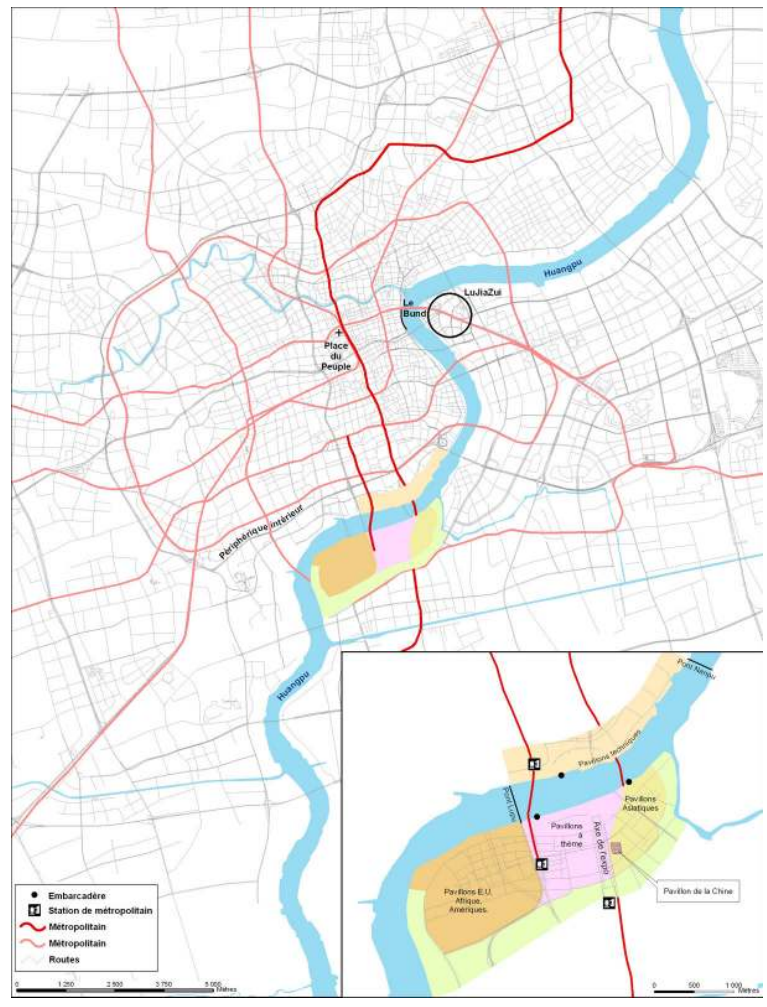

Auteur : Florence Padovani ; conception : Jean-François Cuenot / Prodig, 2010.

9 Ces idées sont exprimées dans le discours officiel : «L'Expo 2010 Shanghai Chine est une fête mondiale organisée par le gouvernement chinois (...) les peuples du monde entier se réuniront à l'Expo Shanghai pour rêver au bel avenir de la «ville ", à partir du thème de cette Expo. $»^{7}$ Ce dernier point est important car il s'agit aussi, et peut-être surtout, d'une fête populaire. 
Illustration 1 - Une horloge à cristaux liquides qui indiquaient les jours restant avant l'ouverture de l'Expo et ensuite les jours depuis son ouverture.

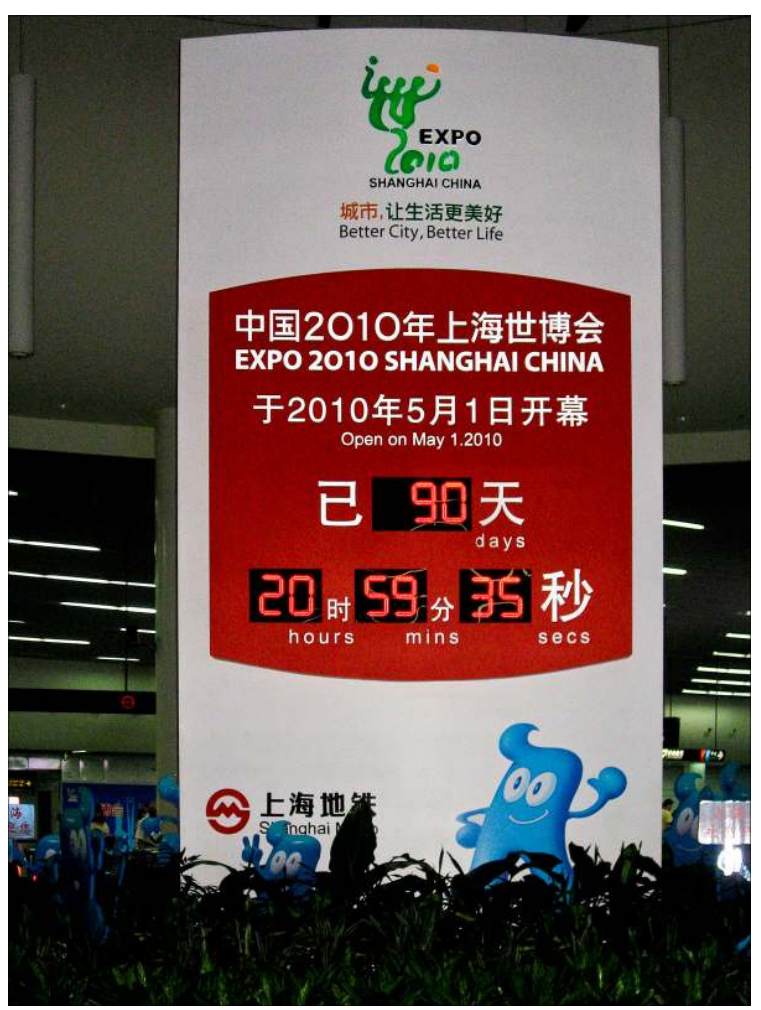

Elle est située dans le métro à la station Place du peuple dans un grand hall que les voyageurs doivent traverser pour changer de ligne de métro.

Auteur : Florence Padovani, 2010.

\section{L'Expo 2010 : une fête populaire}

10 La municipalité de Shanghai s'est fixée comme objectif de faire mieux que les autres villes organisatrices qui l'ont précédée. Le périmètre de l'Expo est vaste puisqu'il s'étend sur 530 hectares de part et d'autre du fleuve Huangpu. Sont attendus « 200 pays et organisations internationales, 70 millions de visiteurs (...) la Chine atteindra ainsi un nouveau record $» .^{8}$ En terme de visiteurs, il est d'ores et déjà certain que leur nombre a fait exploser les records puisque 73 millions de visiteurs ont été enregistrés, dont $95 \%$ de Chinois. Certes l'objectif des cent millions d'entrées n'a pas été atteint mais il s'agit toutefois d'un événement phénoménal. Tous les jours les medias chinois se sont fait le relais des derniers chiffres. Dans les lieux publics et les transports en commun le nombre d'entrées était affiché heure par heure. Si au mois de mai les organisateurs s'inquiétaient de la faible fréquentation (une moyenne de 200000 visiteurs par jour), les mois de juin, juillet et août ont dépassé les 400000 en moyenne9. Fin septembre, plus de 57 millions de visiteurs ont fait le déplacement. Fin août la fréquentation a chuté, ce qui peut s'expliquer par la rentrée des classes et la fin des vacances pour nombre de Chinois.

11 Si la Chine utilise l'Expo comme levier pour faire la promotion de sa modernité et pour confirmer au monde qu'elle fait partie des pays qui comptent sur la scène internationale, il s'agit d'abord de mettre en scène le monde pour un public chinois. Les Jeux Olympiques de Pékin avaient été un grand moment pour la nation chinoise et la municipalité de 
Shanghai entend bien que l'Expo continue de faire vibrer la corde patriotique. Les pavillons des différents pays présents sur le site font d'ailleurs eux aussi, leur propre promotion.

Illustration 2 - Le pavillon de Taïwan

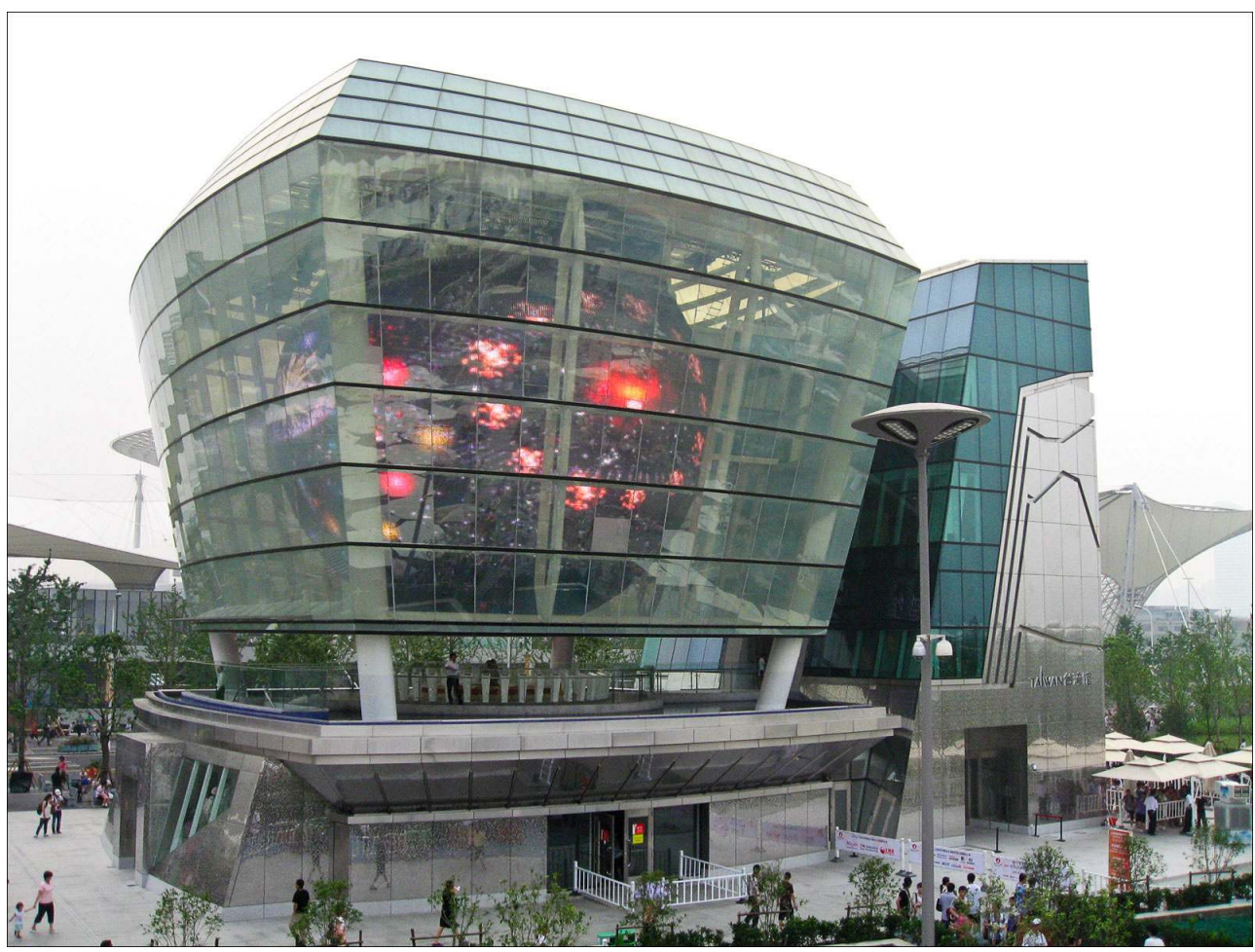

Situé non loin du pavillon de la Chine, il est plus petit, plus moderne dans son architecture. II a attiré de nombreux visiteurs faisant la queue pendant de longues heures.

Auteur : Florence Padovani, 2010

12 S'il n'y a pas d'innovation aussi marquante que le tourniquet permettant de réguler le nombre de personnes entrant (inauguré lors de l'exposition de 1867) ou de bâtiment exceptionnel comme la tour Eiffel, les animations, les spectacles, les illuminations contribuent à une atmosphère digne d'un parc d'attraction. La majorité des Chinois qui viennent des provinces par cars entiers sont déjà enchantés d'être à Shanghai - ville qui attire chaque année nombre de provinciaux. On vient en famille ou avec des collègues de travail, les entreprises organisant des sorties à l'Expo et payant les droits d'entrées pour leur personnel. Les Shanghaïens ont eu droit à un billet d'entrée gratuit par famille, plus une carte de transport (équivalent à 10 euros). Les enfants (moins d'un mètre vingt) ne paient pas, ni les personnes âgées et celles qui les accompagnent. Les personnes âgées et à mobilité réduites n'ont pas besoin de faire la queue. Cette clause donne lieu à un véritable trafic de chaises roulantes aux portes de l'Expo: que ne ferait-on pas pour éviter cinq heures d'attente sous le soleil! 
Illustration 3 - Files d'attente sous le pavillon de la Chine

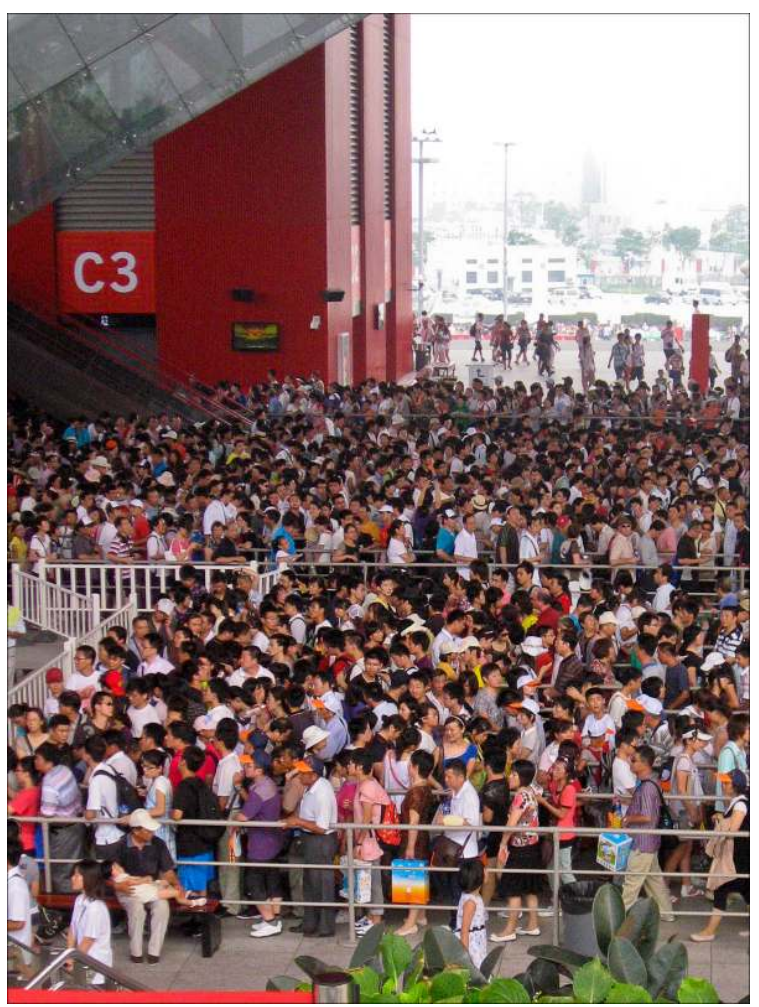

Auteur : Florence Padovani, 2010

Parmi les témoignages recueillis ${ }^{10}$ sur place, la plupart des personnes interrogées se montraient ravies des spectacles africains, intéressées par les pavillons européens et surtout américain mais impressionnées par le pavillon chinois. Bien sûr, les critiques concernant les heures de queue interminables reviennent fréquemment. Il n'était pas rare durant l'été de devoir patienter pendant cinq à six heures pour pénétrer au rez-dechaussée du pavillon chinois. La chaleur éprouvante (plus de $40^{\circ}$ pendant l'été) rendait l'attente d'autant plus pénible et ceci malgré un système de brumisateur et des couloirs couverts pour patienter. En fait, il semble que pour les Chinois qui n'ont pas les moyens financiers de sortir du pays, l'Expo représente une possibilité d'avoir un accès direct à une certaine réalité extérieure. Cette dernière étant bien entendu montrée sous un prisme flatteur et souvent réducteur: la France romantique qui offre des lunes de miel à certains jeunes mariés, l'Italie antique montrant des sculptures romaines, les pays nordiques projetant des petits films ayant trait au ski de fond ou au sauna....

Il s'agit certes d'une fête populaire - et non d'une exposition spécialisée, donc plus technique ou scientifique. Pourtant on peut regretter que, malgré son slogan «meilleure ville, meilleure vie », peu de place ait été laissée aux questions environnementales. Les innovations quant à la ville du futur ou aux moyens de transport non polluants... sont très peu visibles ${ }^{11}$. La zone de la «meilleure pratique urbaine» est située de l'autre côté du fleuve Huangpu, sur Puxi. Il ne s'agit pas de la zone principale de l'Expo. Elle est beaucoup moins développée, considérée comme professionnelle et, bien que nous n'ayons pas les chiffres de fréquentation par zone, il semble qu'elle n'ait pas attiré de grandes foules. La ville écologique de Dongtan (sur lîle de Chongming) qui devait être achevée au moment de l'Expo est restée à l'état de projet. D'autre part, si le pavillon chinois est 
impressionnant par sa taille qui domine tous les autres, par sa couleur rouge qui le met bien en valeur et par sa position au bord de l'axe central, son architecture est très classique. "Ses idées conceptuelles peuvent être résumées comme Couronne orientale, Chine prospère, Grenier du monde, Peuple riche. Sa position spatiale et son orientation montrent les idées de la philosophie orientale sur la relation entre le ciel et la terre. (...) sur la façade en galerie circulaire sont imprimés les noms des dynasties chinoises pour symboliser la longue histoire de la Chine $\${ }^{12}$ Pour les quelques visiteurs pouvant accéder aux étages supérieurs (uniquement sur invitation ou avec un groupe), la leçon d'histoire continue. Un guide explique que le rouleau de peinture animé par des techniques informatiques représente la Chine éternelle ${ }^{13}$, de même que le chariot de bronze emprunté pour l'occasion au musée de la province du Shaanxi. Quant au court montage projeté sur un écran panoramique, il ne cite que des propos de Confucius. A force de faire référence à la "Chine éternelle », on a du mal à voir celle de demain.

Illustration 4 - Le pavillon de la Chine

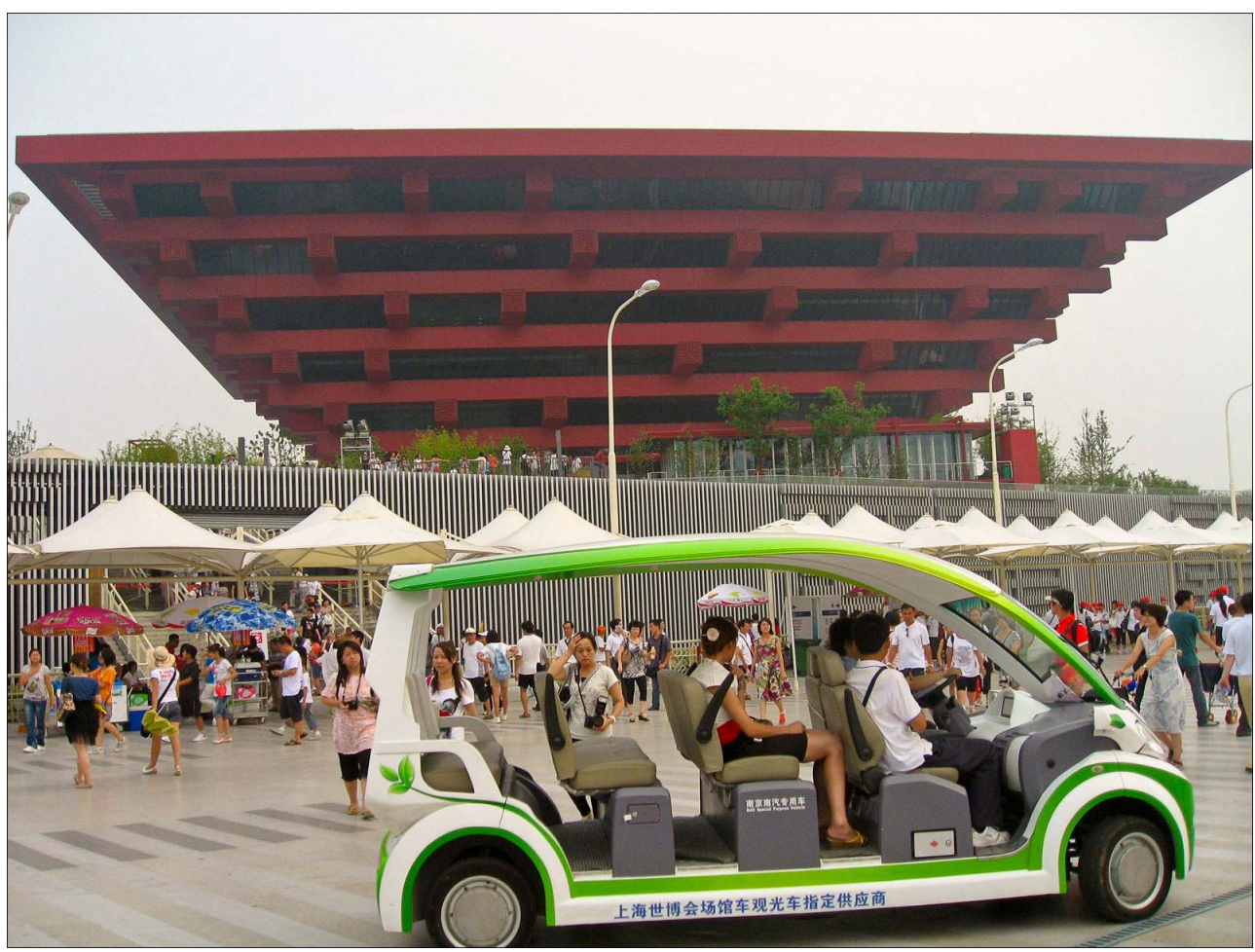

Au premier plan, l'un des moyens de transport électrique utilisé pour sillonner l'Expo.

Auteur : Florence Padovani, 2010.

\section{L'aménagement de la ville}

Pendant plusieurs mois avant l'événement, afin d'accueillir dignement les visiteurs, Shanghai fut en chantier. Pas un seul quartier ne fut épargné par les travaux.

Des restructurations considérables ont été entreprises notamment en matière de transport. L'aéroport de Hongqiao a été agrandi et modernisé, celui de Pudong bénéficie d'un second terminal. Un port accueillant surtout des passagers a été construit dans le prolongement du Bund, en face de la tour de la télévision. Plusieurs autoroutes 
permettant une meilleure intégration au réseau routier de la région (entre la province du Jiangsu, celle du Zhejiang et la municipalité de Shanghai) ont été construites. Shanghai va donc profiter de nouvelles voies de communication régionales et nationales - les autoroutes étant raccordées avec celles menant à Pékin, au Shandong ou les provinces du sud. Les liens dans la région du delta du Yangzi se trouvent encore renforcés. Au niveau de la ville elle-même, en plus de l'extension du réseau de métro déjà existant, la ligne 13 est spécialement dédiée à l'Expo (allant de Madang lu au pont Lupu (site de l'Expo côté Puxi) au terminus Expo - côté Pudong). Un service fluvial desservant le site a lui aussi été mis en place ainsi que des bus. Peu de temps avant l'ouverture de l'Expo l'île de Chongming a été reliée à la terre ferme (Jiangsu d'un côté et Shanghai de l'autre) par un ensemble de ponts et de tunnels. Une partie des usines qui restaient au cœur de la ville ont été délocalisées sur la première île de Changxingxiang.

De même qu'à Pékin, de vieux quartiers de Shanghai ont été soit détruits laissant la place à des immeubles de haut standing, soit transformés en espace culturels, soit démolis et non reconstruits (dans ce cas de grandes affiches cachent le matériel de chantier pour faire meilleure impression). Un certain nombre de sites ayant le label Expo 2010 tentent de retenir les visiteurs pour quelques jours, mais il semble que la majorité des visiteurs arrivent en bus et repartent le jour même ou le lendemain. Les chiffres d'occupation des hôtels ne sont pas encore connus, ni même les retombées économiques pour la ville mais on peut penser que les objectifs ne sont pas remplis. Les hôtels étaient loin d'être pleins et les conférences internationales qui avaient été ajournées pour ne pas causer une trop grande tension sur les capacités d'accueil ont finalement pu avoir lieu.

Illustration 5 - La mascotte de l'Expo (Haibo Trésor de la la mer) et le slogan « Better city, Better life » omniprésents dans la ville avant et pendant la manifestation

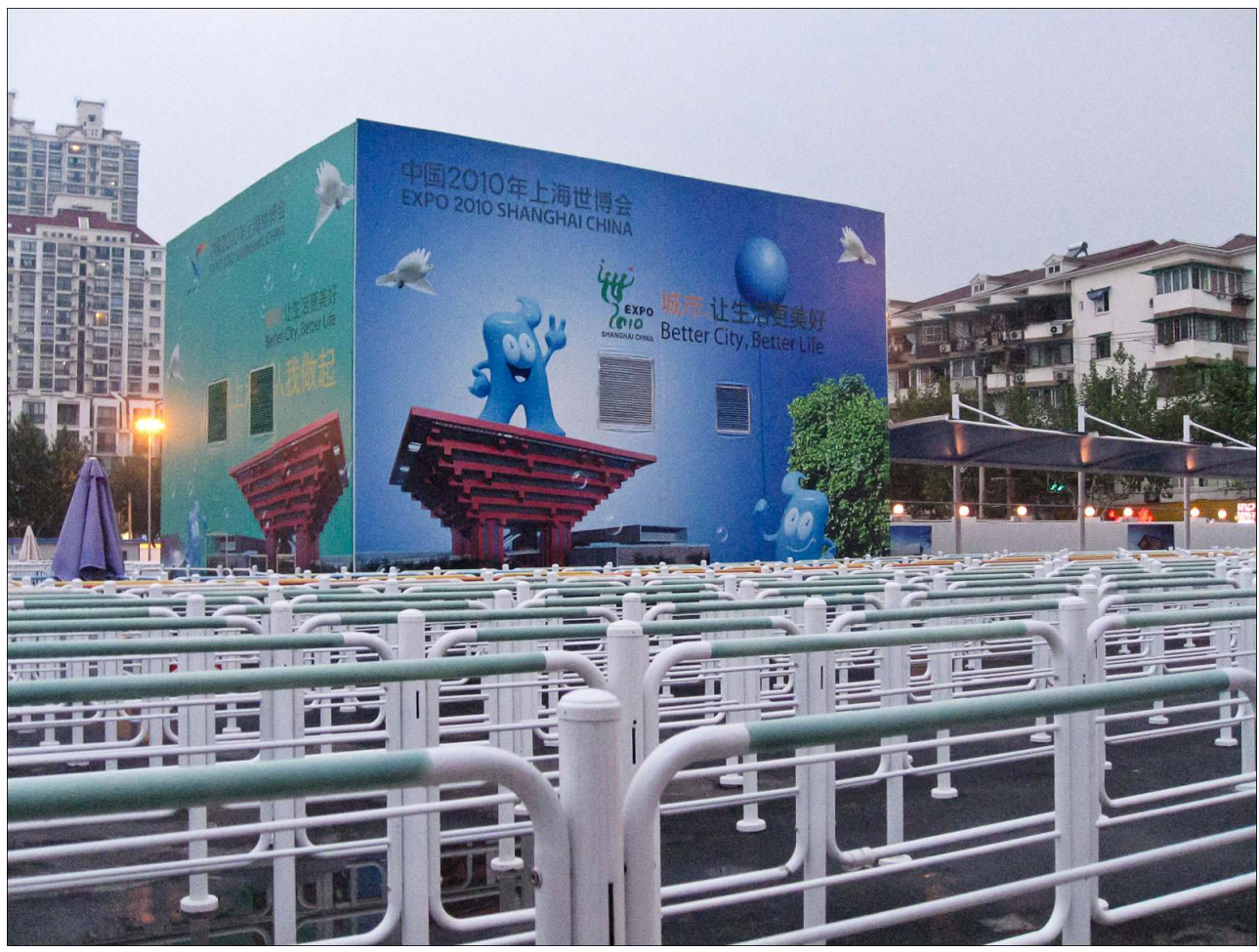

Auteur : Florence Padovani, 2010 
L'exposition universelle de Shanghai a été une grosse opération de communication pour la Chine. Comme les autres pays organisateurs, elle a montré sa puissance et en jouant sur la corde sensible du nationalisme, elle a réalisé une opération de promotion à son profit. Il s'agissait d'un symbole important. Après les Jeux Olympiques, une nouvelle fois, la Chine voulait prouver au monde qu'elle était un partenaire à rang égal avec les pays les plus riches de la planète. Avec son rival japonais, ce sont les deux seuls pays d'Asie à avoir organisé ces deux grands événements internationaux. La fête populaire a été réussie et les Chinois ayant fait le déplacement garderont sûrement un sentiment de fierté nationale. La ville de Shanghai quant à elle n'en sortira peut-être pas bénéficiaire financièrement mais elle aura gagné de nouvelles infrastructures notamment en matière de transport qui resteront après la fin de l'exposition. La ville a aussi bénéficié d'un grand programme de remise à neuf et de rénovation - dont on peut regretter parfois le manque de modération et la précipitation- mais qui a amélioré certains espaces chargés d'histoire comme le Bund.

Peut-être sous la pression de forces globalisantes, d'idées générales acceptées par la majorité des Etats, le discours qui sous-tend l'exposition et la façon de le mettre en scène sont restés très convenus et s'ils sont fédérateurs, à l'échelle de la Chine comme à l'échelle internationale ils n'en manquent pas moins de vision originale et de questionnement. L'ambition affichée par la BIE est d'adresser une question qui affecte « l'ensemble de l'humanité ». Effectivement, les problèmes liés à la ville et au mieux vivre en zone urbaine concerneraient la moitié de la population mondiale.

L'exposition a donc rempli son rôle de communication à usage interne en faisant la promotion d'un nationalisme triomphant. Au niveau international, la communication a très bien fonctionné puisqu'elle a montré l'importance de la Chine et sa capacité à organiser des manifestations de grande ampleur comme une exposition universelle. Par contre, elle n'a pas suffisamment répondu au défi que représente la vie citadine pour la moitié de la population mondiale.

\section{BIBLIOGRAPHIE}

Aperçu sur l'Expo 2010 Shanghai Chine, 2009. Beijing, Groupe d'Edition de Chine.

Convention concernant les Expositions Internationales. http://www.bie-paris.org/site/fr/bie/laconvention.html

Expositions universelles, 2010. Le Monde Dossiers \& Documents, n 399, juillet août 2010.

Hatherley O., 2010. Shanghai expo: this is New Confucianism writ large. guardian.co.uk, Sunday 31 October 2010. http://www.guardian.co.uk/commentisfree/2010/oct/31/shanghai-expoarchitecture-china-development

Houdart S., 2006. Un chaos savamment ordonné...- L'élaboration conceptuelle de l'Exposition internationale japonaise de 2005. Ateliers, $n^{\circ}$ 30, p. 35-62. 
Houdart S., 2003. Exposer la nature d'hier, construire la nature de demain - L'Exposition Internationale japonaise de 2005. Thématique V : «Rapports au corps et à l'environnement », Atelier 14. http://www.reseau-asie.com/

Hugo V., 1867. Introduction au Paris-guide de l'exposition universelle de 1869. Paris, Librairie internationale, chapitre I « L'Avenir ».

Ory P. Les Expositions universelles, de 1851 à 2010 : huit fonctions de la modernité; Conférence mise en ligne par l'Ambassade de France en Chine, http://www.franceshanghai2010.com/ spip.php?article1128

\section{NOTES}

1. Aperçu sur l'Expo 2010 Shanghai Chine, (2009), Beijing, Groupe d'Edition de Chine, p.1.

2. «La présente Convention est ouverte à l'adhésion, d'une part, de tout Etat, soit membre de l'Organisation des Nations Unies, soit non membre de l'ONU qui est Partie au statut de la Cour Internationale de Justice, ou membre d'une institution spécialisée des Nations Unies, ou membre de l'Agence Internationale de l'Energie Atomique et, d'autre part, de tout autre Etat dont la demande d'adhésion est approuvée par la majorité des deux tiers des Parties contractantes ayant droit de vote à l'Assemblée Générale du Bureau. Les instruments d'adhésion sont déposés auprès du Gouvernement de la République Française et prennent effet à la date de leur dépôt». Convention du BIE, art. 35.

3. Convention du BIE, art. 1.

4. http://www.bie-paris.org/site/fr/expos/expo-universelles.html

5. «La multiculturalité, l'ouverture aux autres... C'est le fondement des Expos ! » in Expositions universelles- Le Monde Dossiers \& Documents, 399 - juillet août 2010.

6. «L'Expo est toujours un acte politique » in Le Monde Dossiers \& Documents, op. cité.

7. Aperçu, p. 1.

8. Aperçu, p. 5.

9. http://fr.expo2010.cn/Service/keliutj/index.htm (Site officiel de l'Expo 2010)

10. L'auteur a visité l'Expo à deux reprises en juin et juillet. L'échantillonnage étant réduit (une trentaine de personnes interrogées) nous ne pouvons en tirer de conclusion définitive; nous nous appuyons donc aussi sur les commentaires de collègues et amis chinois ainsi que sur les émissions télévisées, dédiées à l'Expo, suivies pendant l'été.

11. Nous avons mentionné les transports électriques dans l'enceinte de l'exposition. Certains pavillons ont été conçus de manière innovante, tel le bardage qui recouvre le pavillon finlandais, composé de plaques blanches en papier et plastique recyclés. La plupart des pavillons ont d'ailleurs tenté d'intégrer des matériaux recyclables.

12. Aperçu, p. 49-50

13. Il s'agit du Marché à l'époque du festival Qingming - rouleau datant de la dynastie des Song. C'est l'un des tableaux les plus célèbres de la peinture chinoise classique. 


\section{RÉSUMÉS}

L'article explique d'abord le processus d'organisation d'une exposition universelle, avant de montrer comment le montage fait à Shanghai présente plus la Chine face à son histoire et à sa population plutôt que la Chine face au monde. Grande fête populaire « l'Expo Shanghai 2010 » ne fournit que peu de pistes pour l'aménagement des villes du futur.

First the article explains what are the different processes to organize a World Exposition, then it shows that in Shanghai it was done in such a way that the emphasis was more on China facing its own history and its own population rather than China facing the world. "Shanghai Expo 2010 » was a huge popular fair which did not provide many hints for the future urban planning.

\section{INDEX}

Mots-clés : aménagement urbain, exposition universelle, Shanghai

\section{AUTEUR}

\section{FLORENCE PADOVANI}

Florence Padovani est Maître de conférences à l'Université Paris I et membre associée de l'UMR

Prodig. Florence.Padovani@univ-paris1.fr 